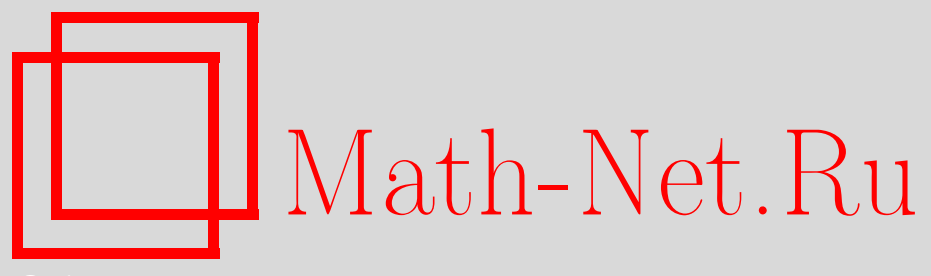

М. Браверман, В. М. Бухштабер, М. Громов, В. Иврий, Ю. А. Кордюков, П. Кучмент, В. Мазья, С. П. Новиков, Т. Сунада, Л. Фридлендер, А. Г. Хованский, Михаил Александрович Шубин (некролог), УМН, 2020, том 75, выпуск 6, 162-170

DOI: https://doi.org/10.4213/rm9968

Использование Общероссийского математического портала Math-Net.Ru подразумевает, что вы прочитали и согласны с пользовательским соглашением http://www . mathnet.ru/rus/agreement

Параметры загрузки:

IP : 54.205 .225 .156

26 апреля 2023 г., 14:58:36

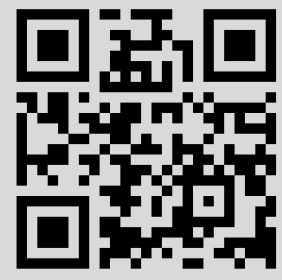




\section{Михаил Александрович Шубин}

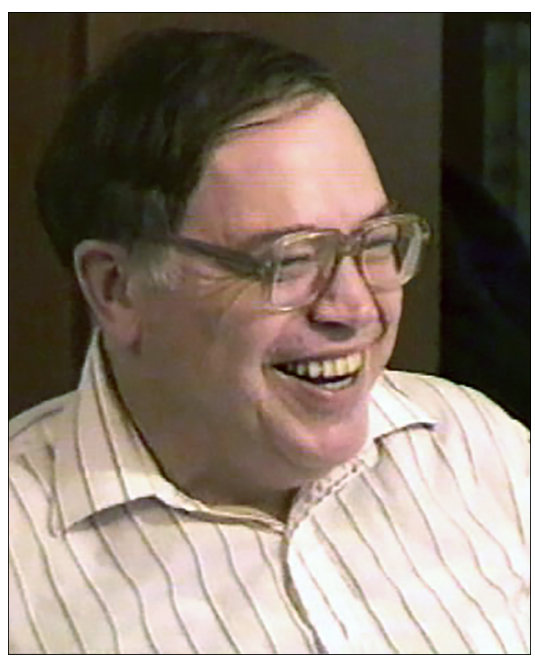

13 мая 2020 г. после длительной болезни скончался замечательный математик Михаил Александрович Шубин. Михаил Александрович родился 19 декабря 1944 г. в Куйбышеве (ныне Самара) и воспитывался мамой и бабушкой. Его мама Мария Аркадьевна работала инженером на Государственном подшипниковом заводе, куда она была распределена в 1941 г. после окончания механико-математического факультете МГУ. Завод в то время был эвакуирован из Москвы в Куйбышев. Мария Аркадьевна проработала на заводе много лет, заведуя лабораторией физики металлов. Впоследствии она защитила кандидатскую диссертацию и перешла в Куйбышевский политехнический институт, где работала доцентом.

В школьные годы основным увлечением М. А. Шубина была музыка. Он обладал абсолютным слухом, окончил музыкальную школу и серьезно думал о поступлении в консерваторию. Однако в старших классах он увлекся математикой, успешно участвовал в олимпиадах и в результате решил поступать на мехмат.

Михаил Александрович поступил на механико-математический факультет МГУ в 1961 г. Когда пришло время выбирать научного руководителя, он пошел в ученики к Марко Иосифовичу Вишику. После окончания мехмата он был принят в аспирантуру и в 1969 г. защитил кандидатскую диссертацию, в которой нашел формулы для вычисления индекса матричных операторов Винера-Хопфа. В частности, для исследования семейств таких операторов ему понадобилось обобщить теорему Бирхго$ф а$, утверждающую, что непрерывная матричнозначная функция $M(z)$ на единичной окружности $|z|=1$ может быть факторизована следующим образом:

$$
M(z)=A_{+}(z) D(z) A_{-}(z),
$$

где $A_{+}(z), A_{-}(z)$ непрерывны и продолжаются аналитически соответственно в единичный круг и в его дополнение (включая бесконечность), а $D(z)$ - диагональная матрица с элементами $z^{n_{j}}$ на диагонали $\left(n_{j}-\right.$ целые числа). М. А. Шубин рассмотрел задачу о том, что происходит, если матрица $M$ непрерывно зависит от дополнительного параметра $t$. Факторизация Бирхгофа не может быть сделана непрерывно по $t$. Михаил Александрович показал, что, если ослабить требование диагональности до треугольной формы, факторизация может быть сделана непрерывной по параметру.

Заслуживает внимания еще одна работа [25] этого периода. Предположим, что семейство замкнутых подпространств некоторого банахова пространства зависит аналитически от многомерного комплексного параметра в том смысле, что локально по

DOI: https://doi.org/10.4213/rm9968 
параметру существует голоморфно зависящий от параметра проектор. Существует ли тогда глобально голоморфный проектор? М. А. Шубин доказал, что если параметр пробегает голоморфно выпуклую область или более общее штейново комплексное пространство, то ответ положителен. Это потребовало применения недавней тогда работы Л. Бунгарта, обобщающей на бесконечномерный случай классическую, трудную (не старую в то время) теорему Грауэрта о совпадении голоморфной и топологической классификаций векторных расслоений над пространствами Штейна. Этот результат М.А. Шубина стал классическим и с тех пор многократно использовался в теории операторов. Он был одним из ранних проявлений широты знаний Михаила Александровича.

В середине 1960-х годов было формализовано понятие псевдодифференциального оператора и началось бурное развитие микролокального анализа, с которым так или иначе будет связана практически вся научная жизнь М. А. Шубина. Совместно с В. Н. Туловским [43] он исследовал спектральную асимптотику оператора $P$ порядка $m>0$ с вещественным вейлевским символом $p(y):=p(x, \xi)$ в $\mathbb{R}^{n}$. Спектр этого оператор дискретен, если выполнены следующие условия:

$$
\left|D^{\alpha} p(y)\right| \leqslant C_{\alpha}(1+|y|)^{m-\rho|\alpha|},
$$

где $0<\rho \leqslant 1$,

$$
\left|D^{\alpha} p(y)\right| \leqslant C_{\alpha} p(y)|y|^{-\rho|\alpha|}
$$

И

$$
p(y) \geqslant a|y|^{m_{0}} \quad \text { при больших }|y|
$$

для положительных $a$ и $m_{0}$. При некоторых дополнительных предположениях на символ они доказали, что количество собственных значений, не превосходящих данного $\lambda$, асимптотически - при $\lambda$, стремящемся к бесконечности - равно $(2 \pi)^{-n}$, умноженному на меру множества $\{y: p(y)<\lambda\}$. Была также получена оценка остатка. Замечателен метод доказательства, который стал потом популярным: используя методы микролокального анализа, авторы построили приближенные спектральные проекторы, а затем применили вариационные методы. Класс операторов с символами, удовлетворяющими оценке (1), часто называется классом Шубина.

В середине и конце 1970-х годов Михаил Александрович в основном занимался операторами с почти периодическими и случайными коэффициентами [26], [27], [29], [31], [32]. Он ввел класс псевдодифференциальных операторов с почти периодическим символом и исследовал спектральные свойства самосопряженных эллиптических операторов из этого класса. Он также доказал существование плотности состояний и энергии Ферми для таких операторов. Пусть $A$ - эллиптический самосопряженный почти периодический дифференциальный оператор в $\mathbb{R}^{n}$ и $\Omega_{k}-$ семейство "хороших" (например, имеющих гладкие границы) возрастающих областей, исчерпывающих все пространство; предположим, что семейство $\Omega_{k}$ "допустимо" в том смысле, что отношение площади границы к объему стремится к нулю при увеличении $k$ (так называемое условие Фёльнера). Рассмотрим в $\Omega_{k}$ оператор $A$ с самосопряженными эллиптическими граничными условиями (например, условиями Дирихле). Пусть $E_{j}(k)-$ его собственные значения, занумерованные в порядке неубывания (с учетом кратности) и $N_{k}(E)$ - количество этих значений, меньших $E$. Плотность состояний - это предел отношения $N_{k}(E) /\left|\Omega_{k}\right|$ при $k \rightarrow \infty$, а энергия Ферми $E^{F}(\rho)$ - это предел последовательности

$$
\frac{1}{p(k)}\left(E_{1}^{(k)}+\cdots+E_{p(k)}^{(k)}\right)
$$

где $k \rightarrow \infty$ и $p(k) /\left|\Omega_{k}\right| \rightarrow \rho$.

В работах [26]-[29], [31], [32], которые составили его докторскую диссертацию, защищенную в 1981 г. в Ленинграде, М. А. Шубин также построил функциональное исчисление псевдодифференциальных операторов с почти периодическими символами, 
установил совпадение спектров в разных функциональных пространствах, доказал аналитическую продолжаемость спектральной $\zeta$-функции и нашел формулу для индекса. В частности, использовался $\mathrm{II}_{\infty}$-фактор, введенный Л. Кобурном, Р. Д. Мойером и И. М. Зингером.

Методы исследования операторов с почти периодическими коэффициентами перекликаются с методами, известными для случайных коэффициентов. Следуя этой связи, М.А. Шубин совместно с Б. В. Федосовым [4] получил формулу для индекса эллиптических операторов в $\mathbb{R}^{n}$ с однородными случайными полями в качестве коэффициентов. Поскольку операторы не являются фредгольмовыми в обычном смысле, индекс определялся в терминах следов в алгебрах фон Неймана. В работах с С. М. Козловым [15], [16] был изучен вопрос о совпадении спектров в разных функциональных пространствах.

М. А. Шубин также внес существенный вклад в теорию операторов с периодическими коэффициентами. Так, в работах с Д. Шенком [23], [24] было получено полное асимптотическое (при энергии, стремящейся к бесконечности) разложение плотности состояний для оператора Хилла с гладким потенциалом. Трудность задачи заключается в том, что в общем положении все лакуны в спектре открыты и тем самым разложение не может быть дифференцируемым. Только недавно многомерный аналог этого результата был получен Л. Парновским и Р. Штеренбергом.

В 1982 г. появилась работа Э. Виттена, где он ввел деформацию комплекса де Рама, ассоциированную с морсовской функцией на замкнутом многообразии, и использовал ее для доказательства неравенств Морса. С. П. Новиков и М. А. Шубин [19]-[21] заметили, что деформация Виттена может быть применена к следующей ситуации. Пусть $M$ - замкнутое многообразие с бесконечной фундаментальной группой и $\widetilde{M}-$ его универсальное накрытие. Снабдим $M$ римановой метрикой, а накрытие $\widetilde{M}$ ее поднятием. Рассмотрим лапласиан $\Delta_{p}$ на $p$-формах на $\widetilde{M}$ и ядро Шварца $K_{p}(x, y)$ ортогонального проектора на ядро $\Delta_{p}$. Фундаментальная группа $\pi_{1}(M)$ действует на $\widetilde{M}$, оставляя функцию $K_{p}(x, y)$ инвариантной. Можно определить $L^{2}$-числа Бетти следующим образом:

$$
\beta_{p}(M):=\int_{F} \operatorname{tr} K_{p}(x, x) d x
$$

где $F$ - фундаментальная область действия $\pi_{1}(M)$ на $\widetilde{M}$. С. П. Новиков и М. А. Шубин доказали, что если $f(x)$ - морсовская функция на $M$, то неравенства Морса сохраняются при замене обычных чисел Бетти на их $L^{2}$-версию. Они также ввели новую систему инвариантов многообразия $M$, впоследствии названных инвариантами Новикова-Шубина. Пусть $N_{p}(\lambda, x, y)$ - ядро Шварца спектрального проектора для оператора $\Delta_{p}$ и

$$
N_{p}(\lambda):=\int_{F} \operatorname{tr} N_{p}(\lambda, x, x) d x .
$$

Инвариант $\alpha_{p}$ - это минимальное число, для которого

$$
N_{p}(\lambda)-\beta_{p}(M)=O\left(\lambda^{\alpha_{p}}\right)
$$

при $\lambda \rightarrow 0$.

С. П. Новиков и М. А. Шубин также ввели понятие фон-неймановской версии кручения Райдемайстера-Рэя-Зингера (см. [20] и замечание после формулы (5) в [19]). Этот инвариант полностью определен, если фон-неймановские числа Бетти равны нулю. Хорошими примерами являются компактные 3-многообразия постоянной отрицательной кривизны. Как выяснилось позднее, в таких случаях логарифм этого инварианта многообразия пропорционален его объему с универсальным ненулевым коэффициентом. 
Позже М. А. Шубин и М. Громов показали [5], что $\alpha_{p}$ являются гомотопическими инвариантами многообразия $M$. В этих работах с Новиковым и Громовым рассматривался также более общий случай представления фундаментальной группы $\pi_{1}(M)$ в $\mathrm{II}_{1}$-факторе.

Классическая теорема Римана-Роха может быть интерпретирована как формула возмущения индекса оператора Коши-Римана при добавлении дивизора нулей и особенностей. Некоторые обобщения этой теоремы на случай других эллиптических операторов были получены в работах В.Г. Мазьи и Б. А. Пламеневского и Н. С. Надирашвили. Далеко идущие обобщения этих теорем для эллиптических дифференциальных операторов в расслоениях над компактными многообразиями и для не обязательно дискретного "дивизора" были получены М. А. Шубиным совместно с М. Громовым [6]-[8]. Мы сформулируем один из их результатов. Пусть $A$ - эллиптический дифференциальный оператор в сечениях векторного расслоения $E$ над компактным многообразием $M, E^{*}$ - двойственное расслоение к $E$ и $A^{*}$ - двойственный оператор. Оснащенным дивизором называется четверка $\left(D^{+}, L^{+} ; D^{-}, L^{-}\right)$, где $D^{+}, D^{-}-$ непересекающиеся замкнутые нигде не плотные подмножества в $M$, а $L^{+}, L^{-}-$конечномерные подпространства пространств распределений со значениями в $E$ и $E^{*}$ соответственно, носители которых лежат соответственно в $D^{+}$и $D^{-}$. Обозначим через $L(\mu, A)$ пространство гладких сечений $E$ над $M \backslash D^{+}$, которые продолжаются до распределений $u$ на $M$ со значениями в $E$ таких, что $u$ ортогонально $L^{-}$и $A u \in L^{+}$. Тогда

$$
\operatorname{dim} L(\mu, A)=\operatorname{ind} A+\operatorname{deg}_{A}(\mu)+\operatorname{dim} L\left(\mu^{-1}, A^{*}\right) .
$$

Здесь $\mu^{-1}:=\left(D^{-}, L^{-} ; D^{+}, L^{+}\right)$и $\operatorname{deg}_{A}(\mu)$ - некая (обычно явно вычисляемая) “степень дивизора", которую мы не будем здесь определять. Заметим, что, переписывая это равенство следующим образом:

$$
\operatorname{dim} L(\mu, A)-\operatorname{dim} L\left(\mu^{-1}, A^{*}\right)=\operatorname{ind} A+\operatorname{deg}_{A}(\mu),
$$

его можно интерпретировать как формулу возмущения индекса оператора при добавлении дивизора "нулей и особенностей".

Позднее М. А. Шубин получил $L^{2}$-версию этого результата для случая некомпактных регулярных накрытий компактных многообразий [37].

Во второй половине 1990-х и в начале 2000-х годов Михаил Александрович много занимался, как сам, так и совместно с В.Г. Мазьей и В.А. Кондратьевым, критериями дискретности спектра и существенной самосопряженности операторов Шрёдингера с электрическим и магнитным потенциалами [11]-[14], [17]. Эти работы связаны со следующим результатом, полученным в 1953 г. А. М. Молчановым: пусть $H=-\Delta+V(x)$ - оператор Шрёдингера в $\mathbb{R}^{n}$ и потенциал $V(x)$ локально интегрируем; А. М. Молчанов показал в 1953 г., что следующее условие эквивалентно дискретности спектра оператора $H$ : для любого $d>0$

$$
\inf \int_{Q_{d} \backslash F} V(x) d x \rightarrow \infty,
$$

когда $Q_{d} \rightarrow \infty$. Здесь $Q_{d}-$ куб с ребрами длины $d$ и гранями, параллельными координатным плоскостям, инфимум берется по компактным подмножествам $F$ таким, что их емкость Винера сар $F$ не превосходит $\gamma$ сар $\overline{Q_{d}}$ (так называемая $\gamma$-пренебрежимость) и $\gamma$ достаточно мало. Отвечая на вопрос И. М. Гельфанда, Мазья и Шубин показали, что любое число $0<\gamma<1$ годится. Более того, можно брать $\gamma$ зависящим от $d$ таким образом, что

$$
\limsup _{d \rightarrow 0} \frac{\gamma(d)}{d^{2}}=\infty .
$$

Для операторов с магнитным потенциалом был введен “эффективный” скалярный потенциал и доказаны теоремы о дискретности спектра в терминах этого потенциала. 
В статье "Can one see the fundamental frequency of a drum?" [18] В. Г. Мазья и М. А. Шубин получили очень красивое и далеко идущее обобщение следующего результата У.К. Хеймана: пусть $\Omega$ - односвязная область в $\mathbb{R}^{2}$ и $r(\Omega)$ - максимальный радиус вписанного в $\Omega$ круга (так называемый внутренний радиус). Тогда минимальное собственное значение оператора Лапласа в $\Omega$ с условиями Дирихле допускает оценку

$$
c r^{-2}(\Omega) \leqslant \lambda_{1}(\Omega) \leqslant C r^{-2}(\Omega)
$$

с абсолютными константами $c$ и $C$. Этот результат неверен для неодносвязных областей (просверливание большого числа малых “дырок” в области дает контрпример), а также в многомерной ситуации. В.Г. Мазья и М.А. Шубин показали, что при некотором изменении понятия внутреннего радиуса результат распространяется на любые области в любой размерности $n$. А именно, пусть $0<\gamma<1$. Определим $r_{\gamma}(\Omega)$ как наибольший радиус замкнутого шара $B_{r}$ такого, что множество $B_{r} \backslash \Omega$ является $\gamma$-пренебрежимым в $B_{r}$. Тогда

$$
c(n, \gamma) r_{\gamma}^{-2}(\Omega) \leqslant \lambda_{1}(\Omega) \leqslant C(n, \gamma) r_{\gamma}^{-2}(\Omega) .
$$

Михаил Александрович занимался и другими разделами математики. Можно отметить, в частности, его незаслуженно малоизвестную работу о псевдоразностных операторах и оценках дискретных функций Грина [34] (см. также [36], [42]). Интерес к дискретным спектральным задачам ныне очень высок, и техника Шубина должна быть крайне полезной. Большое количество его работ (как одиночных, так и совместных с его учениками и соавторами), которые невозможно адекватно отразить в этой статье, относилось к спектральной теории операторов на некомпактных многообразиях и группах Ли (см., в частности, [2], [33], [35], [38]). Он также занимался вполне интегрируемыми задачами [9], [10], применениями нестандартного анализа [44] и пр. Список (возможно, неполный) его работ в базе данных MathSciNet содержит 135 наименований.

М. А. Шубин написал несколько замечательных книг. В 1978 г. вышла его книга "Псевдодифференциальные операторы и спектральная теория" [30], немедленно ставшая одним из основных учебников по микролокальному анализу. Сейчас, более чем через сорок лет после ее выхода, она все еще крайне популярна. Очень влиятельной стала также его совместная с $Ф$.А. Березиным монография "Уравнение Шрёдингера" [1]. В этой книге, законченной уже после смерти Феликса Александровича Березина, изложение многих вопросов, например континуальных интегралов, существенно отличалось от стандартного. В 2001 г. Михаил Александрович написал учебник "Лекции об уравнениях математической физики" [39], в основу которого легли его лекции на экспериментальном потоке мехмата. Несколько расширенный английский перевод этой книги вышел недавно под редакцией М. Бравермана, Р. Макоуэна и П. Топалова [41]. Следует отметить, помимо многочисленных обзоров в УМН, и несколько его фундаментальных обзоров (совместно с другими авторами) в сборниках ВИНИТИ (см., например, [3], [22]). Он также был редактором переводов на русский язык фундаментальных книг $Ф$. Трева и Л. Хёрмандера.

Михаил Александрович стал участником знаменитого семинара И. М. Гельфанда в сентябре 1964 г., будучи студентом IV курса мехмата МГУ. Он вел записи докладов практически без перерыва в течение 25 лет и сохранил все эти записи. Сейчас его конспекты, благодаря финансовой поддержке Института Клея, стали широко доступны в Интернете. ${ }^{1}$ Семинар Израиля Моисеевича Гельфанда был центром математической жизни в СССР и записи докладов, сделанные Шубиным, стали уникальным памятником тех лет.

\footnotetext{
${ }^{1}$ Cм., например, https://www.claymath.org/publications/notes-talks-imgelfand-seminar.
} 
Михаил Александрович был замечательным лектором и педагогом. Его лекции были отработаны до блеска. Он быстро подводил студентов к нетривиальным задачам. Под его руководством защитили диссертации около двадцати аспирантов. Помимо них, он оказал колоссальное влияние на многих других молодых математиков. И не только на студентов и аспирантов вузов, но также на школьников, с которыми он много работал в летних лагерях; одним из результатов этой работы стала публикация его лекций, а также брошюры в серии "Математическое просвещение" [40]. Начиная с 1969 г. М.А. Шубин преподавал на кафедре дифференциальных уравнений мехмата МГУ, в 1992 г. он стал профессором, а позднее заслуженным профессором математического факультета Северо-Восточного университета (Northeastern University) в Бостоне, США.

Михаил Александрович был замечательным интеллигентным, широко образованным, жизнерадостным и доброжелательным человеком. В 1970-х и 1980-х годах он оказывал большую помощь ряду математиков, оказавшихся в трудном положении по политическим причинам. Он также решительно выступал против несправедливостей, имевших место при приеме на мехмат МГУ.

Память о Михаиле Александровиче Шубине, математике, учителе и человеке, навсегда останется в сердцах его многочисленных коллег, учеников и друзей.

М. Браверман, В. М. Бухштабер, М. Громов, В. Иврий, Ю.А. Кордюков, П. Кучмент, В. Мазъя, С.П. Новиков, Т. Сунада, Л. Фридлендер, А.Г. Хованский

\section{Список литературы}

[1] Ф. А. Березин, М. А. Шубин, Уравнение Шредингера, Изд-во МГУ, 1983, 392 с.; англ. пер.: F. A. Berezin, M. A. Shubin, The Schrödinger equation, Math. Appl. (Soviet Ser.), 66, Kluwer Acad. Publ., Dordrecht, 1991, xviii+555 pp.

[2] М.Ш. Браверман, О. Милатович, М. А. Шубин, "Существенная самосопряженность операторов типа Шрёдингера на многообразиях", УМH, 57:4(346) (2002), 3-58; англ. пер.: M. Braverman, O. Milatovic, M. Shubin, "Essential self-adjointness of Schrödinger-type operators on manifolds", Russian Math. Surveys, 57:4 (2002), 641-692.

[3] Ю.В.Егоров, М. А. Шубин, “Линейные дифференциальные уравнения с частными производными. Основы классической теории”, Дифференииальные уравнения с частными производными - 1, Итоги науки и техн. Сер. Соврем. пробл. матем. Фундам. направления, 30, ВИНИТИ, М., 1988, 5-255; англ. пер.: Yu. V. Egorov, M. A. Shubin, "Linear partial differential equations. Foundations of the classical theory", Partial differential equations I, Encyclopaedia Math. Sci., 30, Springer, Berlin, 1992, 1-259.

[4] Б. В. Федосов, М. А. Шубин, "Индекс случайных эллиптических операторов. I", Матем. сб., 106(148):1(5) (1978), 108-140; англ. пер.: В. V. Fedosov, M. A. Shubin, "The index of random elliptic operators. I", Math. USSR-Sb., 34:5 (1978), 671-699.

[5] M. Gromov, M. A. Shubin, "Von Neumann spectra near zero", Geom. Funct. Anal., 1:4 (1991), 375-404.

[6] M. Gromov, M. A. Shubin, "The Riemann-Roch theorem for elliptic operators", I. M. Gel'fand seminar, Adv. Soviet Math., 16, Part 1, Amer. Math. Soc., Providence, RI, 1993, 211-241.

[7] M. Gromov, M. A. Shubin, "The Riemann-Roch theorem for elliptic operators and solvability of elliptic equations with additional conditions on compact subsets" (Saint-Jean-de-Monts, 1993), Journées Équations aux Dérivées Partielles, École Polytech., Palaiseau, 1993, 13 pp. 
[8] M. Gromov, M. A. Shubin, "The Riemann-Roch theorem for elliptic operators and solvability of elliptic equations with additional conditions on compact subsets", Invent. Math., 117:1 (1994), 165-180.

[9] T. Kappeler, P. Perry, M. Shubin, P. Topalov, "The Miura map on the line", Int. Math. Res. Not., 2005:50 (2005), 3091-3133.

[10] T. Kappeler, P. Perry, M. Shubin, P. Topalov, "Solutions of mKdV in classes of functions unbounded at infinity", J. Geom. Anal., 18:2 (2008), 443-477.

[11] V. Kondratiev, V. Maz'ya, M. Shubin, "Discreteness of spectrum and strict positivity criteria for magnetic Schrödinger operators", Comm. Partial Differential Equations, 29:3-4 (2004), 489-521.

[12] V. Kondratiev, V. Maz'ya, M. Shubin, "Gauge optimization and spectral properties of magnetic Schrödinger operators", Comm. Partial Differential Equations, 34:10-12 (2009), 1127-1146.

[13] V. Kondrat'ev, M. Shubin, "Discreteness of spectrum for the Schrödinger operators on manifolds of bounded geometry", The Maz'ya anniversary collection, v. 2 (Rostock, 1998), Oper. Theory Adv. Appl., 110, Birkhäuser, Basel, 1999, 185-226.

[14] V. Kondratiev, M. Shubin, "Discreteness of spectrum for the magnetic Schrödinger operators", Comm. Partial Differential Equations, 27:3-4 (2002), 477-525.

[15] С. М. Козлов, М. А. Шубин, “Теорема о совпадении спектров для случайных эллиптических операторов", Функи. анализ и его прил., 16:4 (1982), 74-75; англ. пер.: S. M. Kozlov, M. A. Shubin, "A theorem on the coincidence of spectra for random elliptic operators", Funct. Anal. Appl., 16:4 (1982), 305-306.

[16] С. М. Козлов, М. А. Шубин, "О совпадении спектров случайных эллиптических операторов", Матем. сб., 123(165):4 (1984), 460-476; англ. пер.: S. M. Kozlov, M. A. Shubin, "On the coincidence of the spectra of random elliptic operators", Math. USSR-Sb., 51:2 (1985), 455-471.

[17] V. Maz'ya, M. Shubin, "Discreteness of spectrum and positivity criteria for Schrödinger operators", Ann. of Math. (2), 162:2 (2005), 919-942.

[18] V. Maz'ya, M. Shubin, "Can one see the fundamental frequency of a drum?", Lett. Math. Phys., 74:2 (2005), 135-151.

[19] С. П. Новиков, М. А. Шубин, "Неравенство Морса и неймановские II $_{1}$-факторы", Докл. АН СССР, 289:2 (1986), 289-292; англ. пер.: S. P. Novikov, M. A. Shubin, "Morse inequalities and von Neumann $\mathrm{II}_{1}$-factors", Soviet Math. Dokl., 34 (1987), 79-82.

[20] С. П. Новиков, М. А. Шубин, "Неравенства Морса и алгебры фон Неймана”, В ст.: "Совместные заседания семинара имени И. Г. Петровского по дифференциальным уравнениям и математическим проблемам физики и Московского математического общества (девятая сессия, 20-23 января 1986 г.)", УМН, 41:4(250) (1986), 163-164.

[21] С. П. Новиков, М. А. Шубин, “Теория Морса и неймановские инварианты неодносвязных многообразий”, В ст.: "Заседания семинара имени И. Г. Петровского по дифференциальным уравнениям и математическим проблемам физики", УМH, 41:5(251) (1986), 222-223.

[22] Г. В. Розенблюм, М.З. Соломяк, М.А. Шубин, “Спектральная теория дифференциальных операторов", Дифференциальные уравнения с частными производными - 7, Итоги науки и техн. Сер. Соврем. пробл. матем. Фундам. направления, 64, ВИНИТИ, М., 1989, 5-242; англ. пер.: G. V. Rozenblyum, M. A. Shubin, M.Z. Solomyak, "Spectral theory of differential operators", Partial differential equations VII, Encyclopaedia Math. Sci., 64, Springer, Berlin, 1994, 1-261.

[23] Д. Шенк, М.А. Шубин, "Асимптотическое разложение плотности состояний и спектральной функции оператора Хилла", Матем. сб., 128(170):4(12) (1985), 
474-491; англ. пер.: D. Schenk, M. A. Shubin, "Asymptotic expansion of the state density and the spectral function of a Hill operator", Math. USSR-Sb., 56:2 (1987), 473-490.

[24] Д. Шенк, М. А. Шубин, “Асимптотическое разложение спектральной функции оператора Хилла", Функи. анализ и его прил., 20:1 (1986), 89-90; англ. пер.: D. Schenk, M. A. Shubin, "Asymptotic expansion of the spectral function of the Hill operator", Funct. Anal. Appl., 20:1 (1986), 78-79.

[25] М. А. Шубин, "О голоморфных семействах подпространств банахова пространства", Матем. исслед., 5:4(18) (1970), 153-165.

[26] М.А. Шубин, “Дифференциальные и псевдодифференциальные операторы в пространствах почти-периодических функций”, Матем. сб., 95(137):4(12) (1974), 560-587; англ. пер.: M. A. Šubin, "Differential and pseudodifferential operators in spaces of almost periodic functions", Math. USSR-Sb., 24:4 (1974), $547-573$.

[27] М. А. Шубин, “Теоремы о совпадении спектров псевдодифференциального почти периодического оператора в пространствах $L^{2}\left(\mathbf{R}^{n}\right)$ и $B^{2}\left(\mathbf{R}^{n}\right)$ ", Cиб. матем. журн., 17:1 (1976), 200-215; англ. пер.: M. A. Shubin, "Theorems on the coincidence of the spectra of pseudodifferential almost-periodic operators in the spaces $L^{2}\left(\mathbf{R}^{n}\right)$ and $B^{2}\left(\mathbf{R}^{n}\right)$ ", Siberian Math. J., 17:1 (1976), 158-170.

[28] М. А. Шубин, "Псевдодифференциальные почти-периодические операторы и алгебры фон Неймана", Тр. ММО, 35, Изд-во Моск. ун-та, М., 1976, 103-164; англ. пер.: M. A. Shubin, "Pseudodifferential almost-periodic operators and von Neumann algebras", Trans. Moscow Math. Soc., 35 (1979), 103-166.

[29] М.А. Шубин, "Почти-периодические функции и дифференциальные операторы с частными производными", УМН, 33:2(200) (1978), 3-47; англ. пер.: M. A. Shubin, "Almost periodic functions and partial differential operators", Russian Math. Surveys, 33:2 (1978), 1-52.

[30] М. А. Шубин, Псевдодифференииальные операторы и спектральная теория, Наука, M., 1978, 279 с.; англ. пер.: M.A. Shubin, Pseudodifferential operators and spectral theory, Springer Ser. Soviet Math., Springer-Verlag, Berlin, 1987, x+278 pp.

[31] М. А. Шубин, "Плотность состояний для самосопряженных эллиптических операторов с почти-периодическими коэффициентами", Тр. сем. им. И. Г. Петровского, 3, Изд-во Моск. ун-та, М., 1978, 243-275; англ. пер.: М. А. Šubin, "The density of states of selfadjoint elliptic operators with almost periodic coefficients", Amer. Math. Soc. Transl. Ser. 2, 118, Amer. Math. Soc., Providence, RI, 1982, 307-339.

[32] М.А. Шубин, "Спектральная теория и индекс эллиптических операторов с почти-периодическими коэффициентами”, УМН, 34:2(206) (1979), 95-135; англ. пер.: M.A. Shubin, "The spectral theory and the index of elliptic operators with almost periodic coefficients", Russian Math. Surveys, 34:2 (1979), 109-157.

[33] М. А. Шубин, "Спектральные свойства и распределение спектра трансверсально эллиптических операторов", Тр. сем. им. И. Г. Петровского, 8, Изд-во Моск. ун-та, М., 1982, 239-258; англ. пер.: M. A. Shubin, "Spectral properties and the spectrum distribution function of a transversally elliptic operator", J. Soviet Math., 32 (1986), 406-422.

[34] М. А. Шубин, "Псевдоразностные операторы и их функция Грина", Изв. АН СССР. Сер. матем., 49:3 (1985), 652-671; англ. пер.: M. A. Shubin, "Pseudodifference operators and their Green's functions", Math. USSR-Izv., 26:3 (1986), 605-622.

[35] M. A. Shubin, "Spectral theory of elliptic operators on non-compact manifolds", Méthodes semi-classiques, v. 1 (Nantes, 1991), Astérisque, 207, Soc. Math. France, Paris, 1992, 35-108. 
[36] M. A. Shubin, "Discrete magnetic Laplacian", Comm. Math. Phys., 164:2 (1994), 259-275.

[37] M. A. Shubin, " $L^{2}$ Riemann-Roch theorem for elliptic operators", Geom. Funct. Anal., 5:2 (1995), 482-527.

[38] M. Shubin, "Spectral theory of the Schrödinger operators on non-compact manifolds: qualitative results", Spectral theory and geometry (Edinburgh, 1998), London Math. Soc. Lecture Note Ser., 273, Cambridge Univ. Press, Cambridge, 1999, 226-283.

[39] М.А. Шубин, Лекции об уравнениях математической физики, МЦНМО, М., 2001, 2003, $303 \mathrm{c}$.

[40] М. А. Шубин, Математический анализ для решения физических задач, Библиотека "Математическое просвещение", 23, МЦНМО, М., 2003, 40 с.

[41] M. Shubin, Invitation to partial differential equations, Grad. Stud. Math., 205, eds. M. Braverman, R. McOwen, P. Topalov, Amer. Math. Soc., Providence, RI, 2020, xvii+319 pp.

[42] M. Shubin, T. Sunada, "Geometric theory of lattice vibrations and specific heat", Pure Appl. Math. Q., 2:3, Special issue: In honor of R. D. MacPherson. Part 1 (2006), $745-777$.

[43] В.Н. Туловский, М.А. Шубин, "Об асимптотическом распределении собственных значений псевдодифференциальных операторов в $\mathbf{R}^{n}$ ", Матем. сб., 92(134):4(12) (1973), 571-588; англ. пер.: V.N. Tulovskiı̆, M. A. Shubin, "On asymptotic distribution of eigenvalues of pseudodifferential operators in $\mathbf{R}^{n}$ ", Math. USSR-Sb., 21:4 (1973), 565-583.

[44] А.К. Звонкин, М.А. Шубин, "Нестандартный анализ и сингулярные возмущения обыкновенных дифференциальных уравнений”, УМH, 39:2(236) (1984), 77-127; англ. пер.: А. K. Zvonkin, M. A. Shubin, "Non-standard analysis and singular perturbations of ordinary differential equations", Russian Math. Surveys, 39:2 (1984), 69-131. 